\title{
Examining Foil Sidewall Damage During TEM Sample Preparation Using Gallium FIB and Needle Geometries
}

\author{
Michael Presley ${ }^{1}$, Dan Huber ${ }^{1}$, Hamish Fraser $^{1}$ \\ ${ }^{1}$ Center for the Accelerated Maturation of Materials, The Ohio State University, Columbus OH
}

The focused ion beam (FIB) has proved itself an invaluable tool for both microscopy and sample preparation. FIBs may be used to create rapidly site specific thin foils that are both highly parallel and thin enough for high resolution electron microscopy. However like all methods for creating thin foils, FIB processing results in artifacts that may obscure features of interest. Of particular interest for electron microscopists is the tendency for thin amorphous layers to develop on FIB produced foils [1], which scatter the incoming electrons, reducing clarity. As these amorphous layers run to the edge of the foil, several authors have had great success showing that a combination of cleaning cross sections, decreasing accelerating voltage, and broad beam argon milling may be used to reduce the degree of amorphization and increase image clarity [2,3]. Unfortunately while the extent of amorphous damage may be reflected in the degree of edge damage, the geometry of a foil precludes direct measurement of the actual thickness of the layers. The amorphous layers lie normal to both the beam and thinnest direction, making it impossible to simply tilt the foil to evaluate the damage. In order to circumvent this obstacle, many authors have resorted to encapsulation in order to measure directly the thickness of the amorphous layer. A trench is made at the locations of interest and subsequently coated with an ex-situ protective layer. The resulting sample must be placed back in the FIB to allow a second foil to be pulled perpendicular to the first. While this method is capable of preserving the damage layers it has two major drawbacks, the first being that the protective layer by its nature modifies the damage surface, and the second being that the method itself is time intensive.

An alternative method for directly measuring the layer thickness is to use a needle geometry. Needles are already being produced by FIB processing for tomography, atom probe, and micro-mechanical testing. Needles and foils both maintain the same orientation with the beam during processing, with the only difference being the curvature of the path of the cleaning cross section. However while the geometry of thin foils precludes direct examination of the damage layer, a needle may be made sufficiently small to be fully electron transparent. The resulting damage layer should be consistent around the entire needle regardless of orientation. Since the surface is unmodified by subsequent coatings and the through thickness view of the needle approximates what is seen in thin foils, a needle may provide a way to directly link the damage layer properties and thickness to image quality.

The needles used in this study were produced using a FEI DualBeam ${ }^{\text {TM }}$ FIB at $30 \mathrm{kV}$ using annular ring patterns with an inner diameter of $150 \mathrm{~nm}$. The needles were then imaged using FEI's Image-Corrected $\operatorname{Titan}^{\mathrm{TM}} \mathrm{G} 2$ 60-300 S/TEM with ChemiSTEM ${ }^{\mathrm{TM}}$ technology. The first needle was extracted from a gold coated single crystal silicon wafer. In Figure 1 the tip of the needle can be clearly seen. The dark region appears fully crystalline in HRTEM while the amorphous layers are both continuous and of equal thickness, confirming the equivalence of the layers around the needle. The amorphous layer measured $\sim 15 \mathrm{~nm}$ along the entire length of the needle, falling well within the range of experimental and modeling work reported for $\mathrm{Si}$ [4]. In HAADF-STEM imaging, the atomic lattice could be detected up to the region of dark contrast in Figure 1, being also $15 \mathrm{~nm}$ in width. By contrast a needle produced from pure aluminum can be seen in Figure 2, where the amorphous layer ranges between 1-3.5 nm in width in both 
HRTEM and HAADF-STEM. Whereas the damage layer in silicon is extremely regular and smooth, the amorphous layer in aluminum appears to be more variable. In each case lattice could be resolved in both HRTEM and HAADF-STEM, providing experimental measurements to be compared with quantitative modeling.
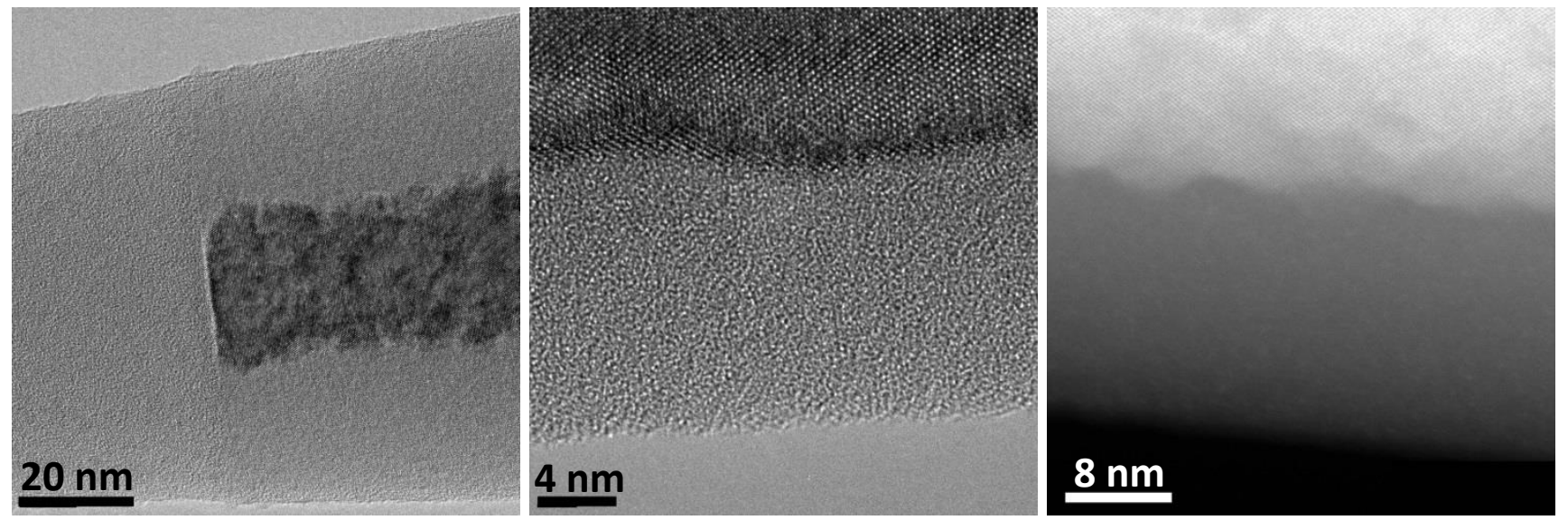

Figure 1. Silicon Needle produced at $30 \mathrm{kV}$ : needle tip HRTEM, amorphous damage layer HRTEM, amorphous damage layer HAADF-STEM
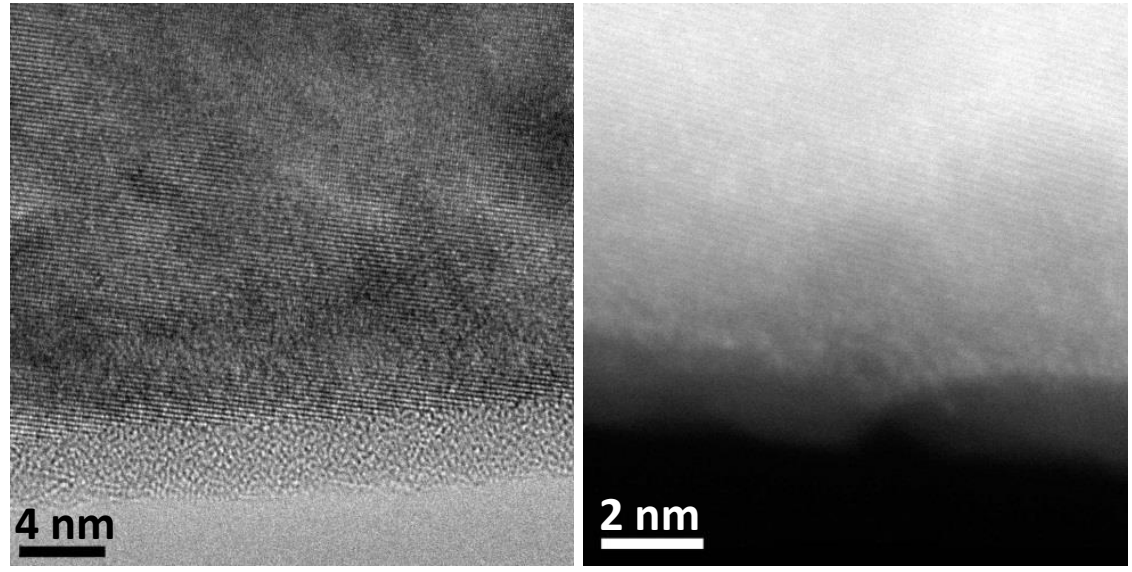

Figure 2. Aluminum Needle produced at $30 \mathrm{kV}$ : amorphous damage layer HRTEM, amorphous damage layer HAADF-STEM

[1] J.P. McCaffrey, M.W. Phaneuf, Ultramicroscopy 87 (2001) 97-104

[2] A. Genc, R.E.A. Williams, D. Huber, H.L. Fraser, Microsc Microanal 13Suppl 2 (2007)

[3] Z. Huang, Journal of Microscopy 215, Pt 3 (2004) 219-223

[4] N. Kato, Journal of Electron Microscopy 53 (2004) 451-458 